\title{
Efficacy of Annona squamosa $L$ in the Synthesis of Glycosaminoglycans and Collagen during Wound Repair in Streptozotocin Induced Diabetic Rats
}

\author{
Thangavel Ponrasu and Lonchin Suguna \\ Department of Biochemistry, Central Leather Research Institute, Council of Scientific and Industrial Research (CSIR), \\ Adyar, Chennai 600 020, India \\ Correspondence should be addressed to Lonchin Suguna; slonchin@yahoo.co.uk
}

Received 26 February 2014; Revised 30 April 2014; Accepted 15 May 2014; Published 9 June 2014

Academic Editor: Ilona Kovalszky

Copyright (C) 2014 T. Ponrasu and L. Suguna. This is an open access article distributed under the Creative Commons Attribution License, which permits unrestricted use, distribution, and reproduction in any medium, provided the original work is properly cited.

\begin{abstract}
The aim of this work was to find out the effects of Annona squamosa on the formation of glycosaminoglycans and collagen during wound healing in normal and diabetic rats. Diabetes induced rats were segregated into 4 groups, each containing six animals. Groups I and III served as the normal and diabetic control while groups II and IV served as normal and diabetic treated. The animals were treated with $200 \mu \mathrm{L}$ of Annona squamosa extract topically. The granulation tissues formed were removed on the 8th day and the amount of glycosaminoglycans (GAGs) and collagen formed was evaluated by sequential extraction and SDSPAGE, respectively. Histological evaluation was also carried out using Masson's trichrome stain. In vitro wound healing efficacy of $A$. squamosa in human dermal fibroblast culture (HDF) was also carried out. The fibroblasts treated with varying concentrations of A. squamosa were examined for proliferation and closure of the wound area and photographed. A. squamosa increased cellular proliferation in HDF culture. The granulation tissues of treated wounds showed increased levels of glycosaminoglycans $(P<0.05)$ and collagen which were also confirmed by histopathology. The results strongly substantiate the beneficial effects of $A$. squamosa on the formation of glycosaminoglycans and collagen during wound healing.
\end{abstract}

\section{Introduction}

Wound healing is the process of repair that follows injury to the skin and other soft tissues. Wound healing cascade begins at the moment of injury or insult and progresses toward complete closure of the wound. Healing requires well-orchestrated integration of the complex biological and molecular events of cell migration, proliferation, and extracellular matrix (ECM) deposition [1]. The distinct interrelated phases which play a crucial role in wound healing are hemostasis, inflammation, proliferation, and remodeling [2]; the end product of wound healing is a dense connective tissue (scar) consisting predominantly of collagen $[3,4]$.

Diabetic wounds are defined as chronic wounds or lesions that take long time to heal or fail to heal [5]. Chronic wounds fail to progress through a normal, orderly, and timely sequence of repair. These wounds may eventually pass through the repair process without restoring sustained anatomical and functional results [6].

Proteoglycans are believed to be involved in the regulation of collagen fibrillogenesis and cell growth and act as tissue organizers [7]. Proteoglycans are the most abundant noncollagenous molecules available in the extracellular matrix, associated with collagen fibrils to form an assembly of the fibrils in vivo, and they may reduce diameter of fibrils by inhibiting lateral growth of fibrils [8]. Glycosaminoglycans (GAGs) are known as collagen associated proteoglycans (PGs) in the extracellular matrix of cell membranes and connective tissues. Glycosaminoglycans and proteoglycans significantly influence the cellular proliferation and rate of wound healing [9].

Proteoglycans, an essential component of mature matrix, are actively synthesized during the proliferative phase of 
wound healing. Fibrin is the primary matrix at the wound site replaced by collagen and proteoglycans gradually [10].

Collagens are the major structural component of the connective tissues, composed of three identical polypeptide chains, each chain comprising Gly-X-Y, where G is glycine and $\mathrm{X}$ and $\mathrm{Y}$ are the imino acids proline and hydroxy proline, respectively. This sequence contributes to the formation of triple helical structure of collagen [11]. Both collagen and glycosaminoglycans play a key role in wound healing. Any agent which affects the normal metabolism of these important connective tissue proteins will definitely affect the normal wound healing pattern [12].

Plant extracts have been widely used as potent wound healing agents throughout the world. The role of plant extracts in glycosaminoglycans and collagen metabolism has been well documented [13, 14].

Annona squamosa L. (Annonaceae), commonly known as custard apple, is a native of West Indies and is cultivated throughout India, mainly for its edible fruit. Phytochemical screening and efficacy of this plant on wound healing in diabetic rats have already been reported by us [15].

In this paper, we report the influence of $A$. squamosa extract on the synthesis and characterization of glycosaminoglycans and collagen with detailed histological analysis during wound healing in streptozotocin-nicotinamide induced diabetic rats. This study also explores the role of $A$. squamosa in fibroblast proliferation with respect to wound closure rate.

\section{Materials and Methods}

2.1. Plant Collection and Extraction. A. squamosa leaves were procured, shade-dried, and crushed to make fine powder. $100 \mathrm{~g}$ of this powder was macerated with $70 \%$ ethanol in dark and filtered to harvest a viscous supernatant. The extract was dried under vacuum below $40^{\circ} \mathrm{C}$. The viscous residue was collected, weighed, and kept at $4^{\circ} \mathrm{C}$ until use. The extract was reconstituted in phosphate buffered saline (PBS) whenever required .

2.2. Chemicals. D-Glucuronic acid, chondroitin sulphate $\mathrm{B}$, chondroitinase ABC, chondroitinase AC, Streptomyces hyaluronidase, $\beta$-D-galactosidase, sodium dodecyl sulphate, coomassie blue R-250, N,N-methylene bisacrylamide, and B-mercaptoethanol were purchased from Sigma Chemical Company, St. Louis, USA. Pepsin, proteinase K, and potassium acetate were procured from Sisco Research Laboratory, Mumbai, India. All other reagents were of high analytical grade.

\subsection{In Vitro Studies}

2.3.1. MTT Assay. For quantitative evaluation of cell viability and proliferation, MTT (3-[4,5-dimethylthiazol-2-yl]-2,5diphenyl tetrazolium bromide) assay was used, in which only viable cells can reduce MTT to insoluble purple formazan [16]. Thus, the intensity of purple color represents the number of viable cells. For MTT assay, the HDF cells were cultured in 24-well microtiter plate at a density of $5 \times 10^{3}$ cells per
$100 \mu \mathrm{L}$ for $48 \mathrm{~h}$. The culture medium was supplemented with different concentrations of $A$. squamosa like 7.8, 15.6, 31.2, $62.5,125,250,500$, and 1000 parts per million (ppm). The cells were then incubated in a humidified atmosphere of $5 \%$ $\mathrm{CO}_{2}$ at $37^{\circ} \mathrm{C}$ and medium was changed every day (wherever applicable). MTT assay was performed at $48 \mathrm{~h}$ after treatment. The formazan crystals formed by living cells were solubilized with DMSO and the absorbance was measured at $570 \mathrm{~nm}$ with background subtraction at $690 \mathrm{~nm}$ using multimode plate reader (TECAN, Infinite $M$ 200).

2.3.2. In Vitro Wound Healing Assay. The in vitro wound healing assay was carried out as previously described by Kheradmand et al. [17]. HDF cells were seeded on 24-well tissue culture dishes $\left(10^{6}\right.$ cells/well). The cells were incubated for $48 \mathrm{~h}$ at $37^{\circ} \mathrm{C}$ with $5 \% \mathrm{CO}_{2}$. When confluence was reached, cell monolayers were incubated for $12 \mathrm{~h}$ in serumfree medium. The monolayers were then gently scratched with a sterile pipette tip and extensively rinsed with medium to remove all cellular debris. An indicated concentration of plant extract was added and incubated for 24,48 , and $72 \mathrm{~h}$. The average extent of wound closure was evaluated by measuring the width of the wound. The rate of proliferation of cells into the wound area was examined by placing the cells under an inverted microscope and the image obtained was photographed.

\subsection{In Vivo Studies}

2.4.1. Experimental Design and Diabetes Induction. Healthy male Wistar albino rats (150-200 g) were used for the in vivo wound healing study. The rats were fed with commercial rat feed and water ad libitum. All clinical procedures were carried out according to the guidelines of the Institutional Animal Care and Use Committee (IACUC). A formal approval from the Animal Ethical Committee has also been obtained.

Diabetes was induced by a single intraperitoneal injection of streptozotocin $(50 \mathrm{mg} / \mathrm{kg} \mathrm{b} \cdot \mathrm{wt})$ dissolved in $0.1 \mathrm{M}$ of cold citrate buffer ( $\mathrm{pH} 4.5$ ), 15 min after the intraperitoneal administration of nicotinamide $(110 \mathrm{mg} / \mathrm{kg} \mathrm{b} \cdot \mathrm{wt})$ in overnight fasted rats $[18,19]$. Diabetic status was confirmed by tail vein blood glucose estimation using glucometer (One Touch Horizon, Johnson \& Johnson, Mumbai, India) after $72 \mathrm{~h}$. Two weeks after diabetic induction, rats with blood glucose level $>250 \mathrm{mg} \mathrm{dL}^{-1}$ were deemed diabetic and used for the experiment.

The rats were divided into four groups comprising six rats in each group as given below:

group I: control rats left untreated,

group II: rats treated with $A$. squamosa $(200 \mu \mathrm{L})$ at a concentration of $(100 \mathrm{mg} / \mathrm{kg} \mathrm{b} \cdot \mathrm{wt})$,

group III: diabetic control rats also left untreated,

group IV: diabetic rats treated with $A$. squamosa $(200 \mu \mathrm{L})$ at a concentration $(100 \mathrm{mg} / \mathrm{kg} \mathrm{b} \cdot \mathrm{wt})$.

2.4.2. Excision Wound and Drug Administration. Wounds were created after the confirmation of diabetes. Rats were 
anaesthetized by the intraperitoneal injection of thiopentone $(50 \mathrm{mg} / \mathrm{kg} \mathrm{b} \cdot \mathrm{wt})$ dissolved in sterile distilled water [20]. A $2 \mathrm{~cm}^{2}(4 \mathrm{~cm})$ full thickness open excision wound was made on the back of the rat as reported in our earlier studies [15]. The control rats were left untreated and the treated rats were administered once daily with $200 \mu \mathrm{L}(100 \mathrm{mg} / \mathrm{kg}$ b.wt reconstituted in PBS) of the extract for 8 days. The animals were sacrificed and the wound tissues were removed on day 8 after wounding and used for the glycosaminoglycan and collagen analyses.

2.4.3. Glycosaminoglycans (GAGs). Total GAGs from the wound tissues were extracted as described by Smith et al. [21]. The amount of GAGs was determined by estimating uronic acid content [22]. Individual GAGs were estimated using GAG degrading enzymes and nitrous acid treatment as described by Breen et al. [23].

2.4.4. Collagen. Fractionation of collagen was performed by the method of Piez [24]. The susceptibility of insoluble collagen to denaturing agents like urea and potassium thiocyanate was analyzed by the method of Adam et al. [25]. The aldehyde content of the acid soluble collagen was estimated according to the method of Paz et al. [26].

2.4.5. SDS-PAGE. The subunit composition of the isolated collagen was investigated by sodium dodecyl sulfatepolyacrylamide gel electrophoresis (SDS-PAGE) [27]. Briefly, collagen bands were separated by SDS-PAGE using 3\% stacking gel with $5 \%$ separating gel and Coomassie brilliant blue staining. Interrupted SDS-PAGE was used for the separation of type III collagen.

2.5. Histopathology. The rats were sacrificed and the tissues were removed from the wound site periodically. These samples were then separately fixed in $10 \%$ formalin-saline, dehydrated through graded alcohol series, cleared in xylene, and embedded in paraffin wax (melting point $56^{\circ} \mathrm{C}$ ). Serial sections of $5 \mu \mathrm{m}$ were cut and stained with Masson's trichrome. The sections were examined under light microscope and photomicrographs were taken for the analysis.

2.6. Statistical Analysis. Data were expressed as mean $\pm \mathrm{SD}$ of six animals in each group and the results were statistically evaluated using one-way ANOVA and Student's paired $t$ test. All statistical analyses were performed using GraphPad prism (version 5.0; GraphPad software Inc., San Diego, CA, USA). Values corresponding to $P<0.05$ were considered as significant.

\section{Results}

3.1. Cell Viability Assay. The proliferation and viability of the cells in the presence of different concentrations of the extract (7.8-1000 ppm) were observed for $48 \mathrm{~h}$, by MTT assay. A significant increase in the cell viability was observed up to a concentration of $125 \mathrm{ppm}$ as depicted in Figure 1. The

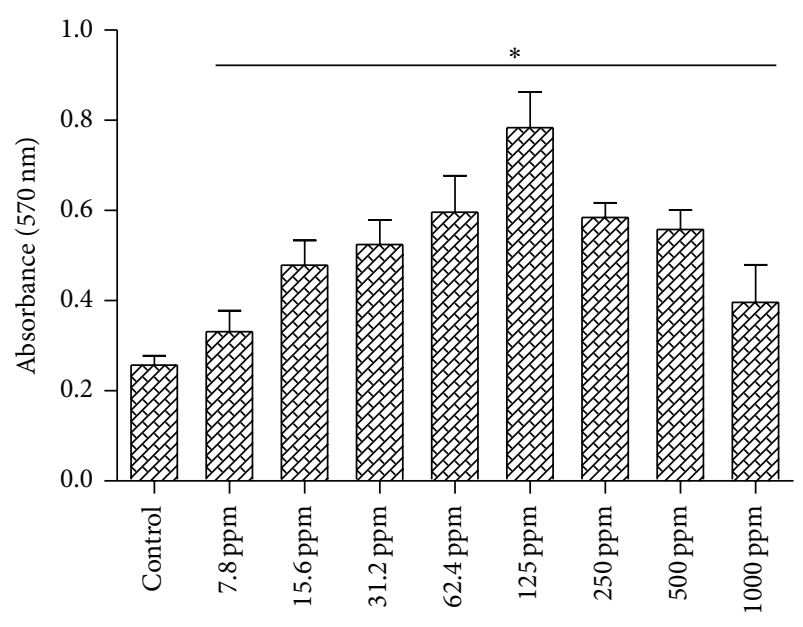

$48 \mathrm{~h}$

FIgURE 1: Efficacy of A. squamosa extract on the proliferation of HDF cells for $48 \mathrm{hrs}$. HDF cells were cultured with graded concentrations of A. squamosa extract (7.8 ppm-1000 ppm) and the number of viable cells was determined by MTT assay after $48 \mathrm{hrs}$. The results revealed that the $A$. squamosa extract increased the viable cells with increasing concentrations and there is no toxic or adverse effect at any concentration. All the data were expressed as mean \pm $\mathrm{SD}(n=6)$ and statistically significant $\left({ }^{*} P<0.05\right)$ when compared to control culture.

proliferation was found to increase within the same cells day by day (data not shown). Culture supplemented with high concentrations like 250,500 , and $1000 \mathrm{ppm}$ showed slightly lesser number of viable cells than the concentrations up to $250 \mathrm{ppm}$. Based on this, concentrations between 7.8 and $125 \mathrm{ppm}$ of $A$. squamosa extract were used for further study.

3.2. Efficacy of A. squamosa in In Vitro Wound Healing. In vitro wound healing assay was carried out using scratch wound model in confluent HDF culture. The proliferation and wound closure were evaluated until the completion of healing. After making the scratch, the cells were supplemented with $7.8-125 \mathrm{ppm}$ of A. squamosa extract and photomicrographs were taken using inverted microscope to assess wound healing at different time point intervals. Figure 2 shows the images of the scratch wounds treated with $125 \mathrm{ppm}$ of $A$. squamosa, taken on days 0,1 , and 2 , as this concentration showed the best results. A. squamosa supplementation showed enhanced proliferation of HDF cells and wound closure was completed within two days, whereas part of incomplete wound was observed in control culture and it took almost three days to complete healing. For each concentration and each time frame, three scratched wells were used to assess wound healing. These results suggested that $A$. squamosa has no cytotoxic effect and has significant effect on proliferation of HDF cells in in vitro, which would be advantageous for reepithelialization during wound healing.

3.3. Effect of A. squamosa on Glycosaminoglycans. Levels of total GAGs evaluated on 8th day wound tissues of 

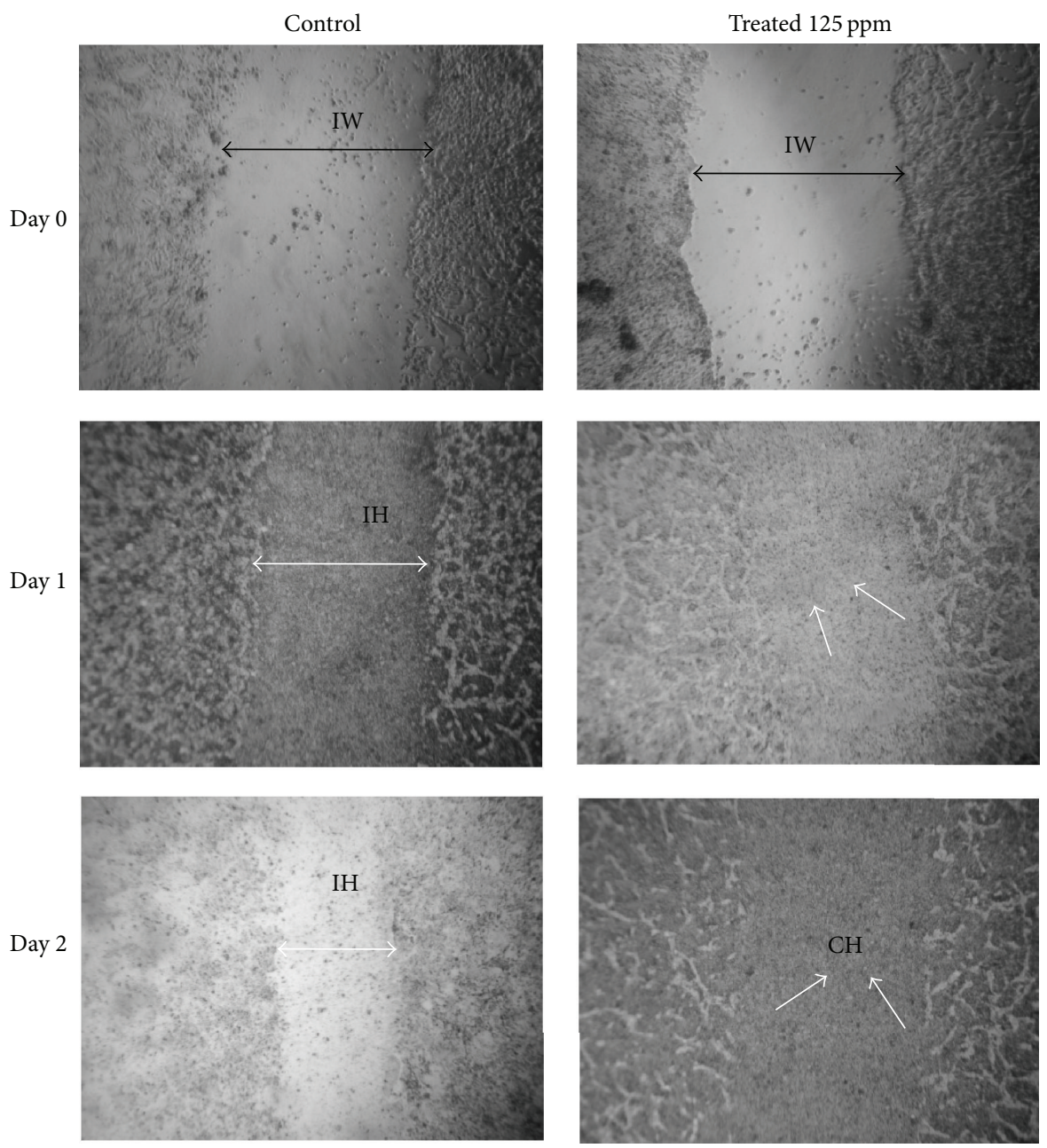

Figure 2: In vitro wound healing of HDF cells of control culture and A. squamosa treated (supplemented with 7.8-125 ppm) cultures. Proliferation of HDF cells was observed for 2 days after wound creation. The double headed black arrow shows the margin of scratched area on day 0 as initial wound (IW), and double headed white arrow shows the incomplete healing (IH), whereas white arrow shows the complete healing $(\mathrm{CH})$. A. squamosa treatment shows complete healing on day 2 , which clearly indicates that the A. squamosa accelerates the rate of proliferation faster in treated culture than the control.

the control and treated rats are shown in Figure 3. The values are expressed as uronic acid equivalence. Synthesis of ground substances was found to be high in treated groups. The amount of total GAGs was significantly $(P<0.05)$ higher (53\%) in normal treated (group II) than control. In group IV, the increase was around $46 \%$ when compared to group III diabetic control (Figure 3(a)). Percentage of individual GAGs determined in the wound tissues of control and $A$. squamosa treated groups is shown in Figure 3(b). Among the various GAGs formed in the wound tissues, hyaluronic acid levels have a major proportion in both untreated and treated groups. In normal treated group, about $11 \%$ increase was observed when compared to control. Similar trend (8\%) was observed in diabetic treated group for hyaluronic acid. A $7 \%$ and a $6 \%$ increase in chondroitin sulfates were observed in normal treated and diabetic treated groups as compared to their respective controls. Dermatan sulfate was also significantly increased in normal treated group (7\%) and diabetic treated group (11\%) when compared to control.
But, keratan sulfate content was significantly reduced in both diabetic treated $(29 \%)$ and normal treated groups (40\%) when compared to their respective controls.

3.4. Effect of A. squamosa on Collagen. Table 1 illustrates the solubility pattern of collagen of day 8 tissues of control and treated rats in terms of neutral salt soluble (NSS), acid soluble (AS), and pepsin soluble (PS) collagen and insoluble collagen (IS). In all groups, pepsin digestion showed a significantly higher amount of collagen particularly in A. squamosa treated rats. The amount of AS fraction was greatly improved in $A$. squamosa treated groups. The difference between normal and diabetic treated group was found to be $18 \%$. A similar kind of trend was observed for IS in treated groups. It shows that there was abundant and earlier maturation of collagen fibers in A. squamosa treatment.

The susceptibility AS to different denaturing agents like $2 \mathrm{M}$ KCNS and $6 \mathrm{M}$ urea was carried out in both control 
TABLE 1: Effect of A. squamosa on solubility pattern of collagen on day 8 wound tissues.

\begin{tabular}{lcccc}
\hline Group & Neutral salt soluble collagen & Acid soluble collagen & Pepsin soluble collagen & Insoluble collagen \\
\hline Control & $84.5 \pm 10.4$ & $590.5 \pm 42.4$ & $2289 \pm 187.5$ & $976.7 \pm 10.2$ \\
Treated & $97.8 \pm 9.6$ & $898.8 \pm 86.5^{*}$ & $2812 \pm 195.8^{*}$ & $1039.8 \pm 17.4^{*}$ \\
Diabetic control & $64.9 \pm 8.2$ & $335.5 \pm 66.1$ & $1467 \pm 173.4$ & $841.3 \pm 7.0$ \\
Diabetic treated & $55.3 \pm 9.2$ & $465.0 \pm 62.6^{*}$ & $1766 \pm 90.4^{*}$ & $883.7 \pm 13.2$ \\
\hline
\end{tabular}

Values ( $\mu \mathrm{g} / 100 \mathrm{mg}$ wet tissue) are expressed as mean $\pm \mathrm{SD}(n=6$ animals).

${ }^{*} P<0.05$ is considered as significant compared to corresponding control.

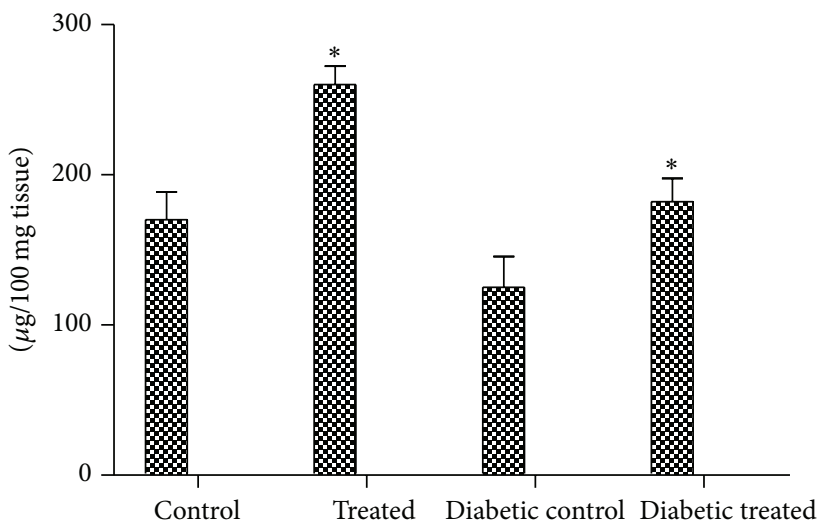

(a)

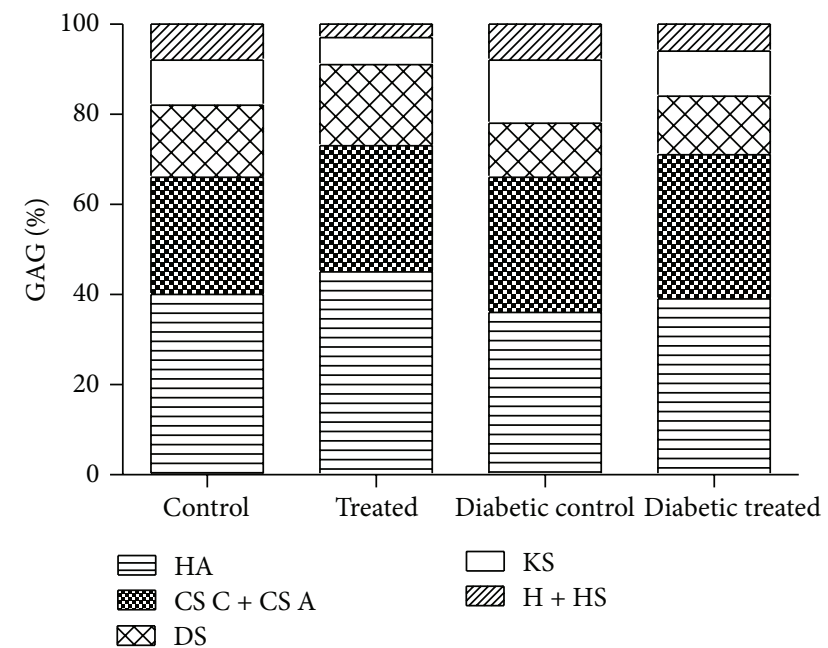

(b)

Figure 3: (a) Glycosaminoglycans of control and A. squamosa treated wound tissues on day 8 after wounding. Values are expressed as mean \pm SD for six animals. ${ }^{*} P<0.05$ is considered as significant compared with the control. (b) Percentage proportions of various glycosaminoglycans formed in control and A. squamosa treated wound tissues on day 8 after wounding. HA: hyaluronic acid; CS C + CS A: chondroitin sulphate A and C; DS: dermatan sulphate; KS: keratan sulphate; H + HS: heparin and heparin sulphate.

and treated wound tissues. The amount of collagen released by these agents was significantly $(P<0.05)$ reduced in $A$. squamosa treated rats. $2 \mathrm{M}$ KCNS treatment showed $46 \%$ release for control and 33\% for extract treated AS. Similarly, $41 \%$ and $34 \%$ of decreased collagen release were obtained for both diabetic control and diabetic treated (Table 2).

A. squamosa treated wound tissues showed high aldehyde content in the pepsin soluble collagen. There were about $46 \%$ increase in normal and $38 \%$ increase in diabetic treated groups when compared to control (Table 3). The increase in aldehyde content and reduction of susceptibility pattern in $A$. squamosa treated rats significantly explain the formation and maturation of collagen.

3.5. SDS-PAGE. Figure 4(a) shows the SDS-PAGE pattern of pepsin soluble collagen from control and treated wound tissues. From the banding pattern, we could observe a significant increase in type I collagen in A. squamosa treated normal and diabetic rats. Figure 4(b) depicts the interrupted gel electrophoresis of pepsin soluble collagen, from which we could observe a marked increase in type III collagen in the treated groups.
TABLE 2: Effect of A. squamosa on susceptibility of insoluble collagen on day 8 wound tissues.

\begin{tabular}{lcc}
\hline Group & $2 \mathrm{M} \mathrm{KCNS}$ & $6 \mathrm{M}$ urea \\
\hline Control & $5.75 \pm 0.79$ & $6.70 \pm 0.56$ \\
Treated & $3.08 \pm 0.58^{*}$ & $3.98 \pm 0.63^{*}$ \\
Diabetic control & $3.49 \pm 0.79$ & $3.47 \pm 0.79$ \\
Diabetic treated & $2.33 \pm 0.76^{*}$ & $2.29 \pm 0.69^{*}$ \\
\hline
\end{tabular}

Values (mg/100 mg collagen) are expressed as mean \pm SD ( $n=6$ animals). ${ }^{*} P<0.05$ is considered as significant compared to corresponding control.

TABLE 3: Aldehyde content of acid soluble collagen on day 8 wound tissues of control and A. squamosa treated rats.

\begin{tabular}{lc}
\hline Control & $4.59 \pm 1.01$ \\
Treated & $8.51 \pm 1.09^{*}$ \\
Diabetic control & $3.22 \pm 0.90$ \\
Diabetic treated & $5.20 \pm 1.08^{*}$ \\
\hline
\end{tabular}

Values ( $\mu \mathrm{M} / 100 \mathrm{mg}$ collagen) are expressed as mean $\pm \mathrm{SD}$ ( $n=6$ animals). ${ }^{*} P<0.05$ is considered as significant compared to corresponding control. 


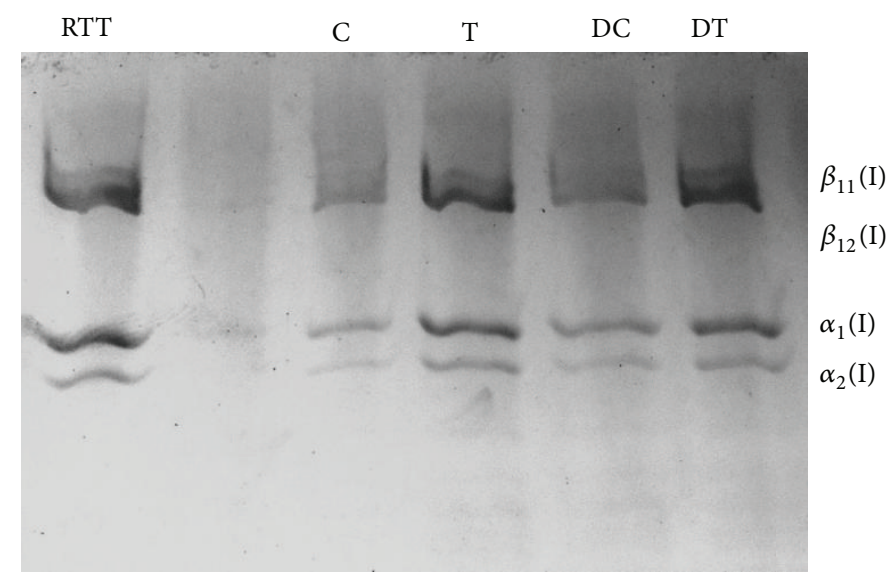

(a)

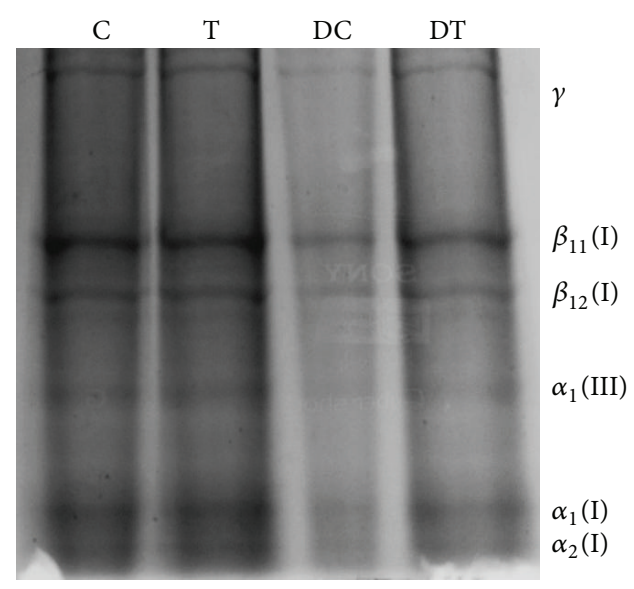

(b)

FIGURE 4: Normal (a) and interrupted (b) SDS-PAGE photograph showing the distribution of collagen. All lanes were loaded with equal concentration of collagen $(25 \mu \mathrm{g})$. RTT: rat tail tendon; C: control; T: treated; DC: diabetic control; DT: diabetic treated.

3.6. Histopathology of Wound Tissues. A detailed histological evaluation was carried out to screen the collagen maturation using Masson's trichrome staining. Masson's trichrome stained wound tissue of the first week normal control showed less collagen fibers, proliferating blood capillaries, and incomplete epithelialization (Figure 5(a)). Whereas, complete fibrous tissue, more number of collagen bundles and large number of blood vessels were observed in treated tissue (Figure 5(b)). In the second week, the amount of collagen deposited on the wound surface in control tissue was found to be inadequate (Figure 5(c)). But, more accumulation of collagen fibers was seen in treated tissue (Figure 5(d)).

In diabetic group, the first week of control tissue showed large number of blood vessels with minimal cellular infiltrates on the wounded site (Figure 6(a)). In treated tissue, large amount of collagen has been observed with an early epithelialization process (Figure 6(b)). The second week of control tissue showed equal amount of blood capillaries and collagen bundles at the wound site (Figure 6(c)). Thick collagen fiber deposition under the completed epithelial layer on the wound surface was seen in treated tissue (Figure 6(d)). In the third week, fewer amounts of loosely packed collagen molecules were accumulated in untreated tissue depicting incomplete wound closure (Figure 6(e)). But, highly organized thick collagen fibers as bundles and thick uniform epithelial layer were formed in treated tissue (Figure 6(f)).

\section{Discussion}

In this investigation, efficacy of $A$. squamosa extract in in vitro cell viability, proliferation of HDF cells, and its wound closure was studied to confirm the therapeutic activity of this extract in wound healing.

Even though various models have been proposed to study reepithelialization process in in vitro, the simple one is fibroblast monolayer scratch wound, in which injury was mechanically created in confluent fibroblast culture using sterile microtip and recovery of wound area has been used as an indicator for wound reepithelialization [28]. After scratch wounding, the neighboring intact mesenchymal cells start to migrate rapidly over the wounded area until they cover the wound surface with cells. Then the cells begin to proliferate to increase number of viable cells that restore the normal epithelial structure. Later, the fibroblasts start to differentiate on the wounded surface area of the healed site and become soft and smooth, and a well-layered architecture is restored [29]. In our results, we found that all the concentrations of $A$. squamosa extract (7.8 ppm-1000 ppm) increased the number of viable cells and proliferation of fibroblasts and cover the wound surface rapidly. There is no significant toxicity and adequate antiproliferative effect observed in A. squamosa treatment.

Cell proliferation is an essential event during reepithelialization, so proliferating fibroblasts at the wound site ensure an adequate supply of cells to migrate and cover the wound surface. Synthesis of extracellular matrix (ECM) is a key feature of wound healing. Dermal reconstruction is characterized by the formation of granulation tissue, which includes cell proliferation, ECM deposition, wound contraction, and angiogenesis [30].

After tissue injury, synthesis of ground substances plays an important role during wound healing process. They are mainly PGs and GAGs. GAGs have been found to be regulators of cellular proliferation, migration, and differentiation [31]. Synthesis of these substances and their degradation has great impact on the healing process. The GAGs are the first components of the extracellular matrix to be synthesized during wound healing and form the scaffold for collagen and elastin deposition [32]. Our results revealed that A. squamosa extract significantly improved the total GAGs in normal and diabetic treated rats more than the control.

HA, a known glycosaminoglycan, is an important physiological substance that plays a vital role in the healthy formation of connective tissue [33]. HA has also been shown to stimulate collagen synthesis in fetal fibroblast cultures [34]. Fibroblasts are the major components which 


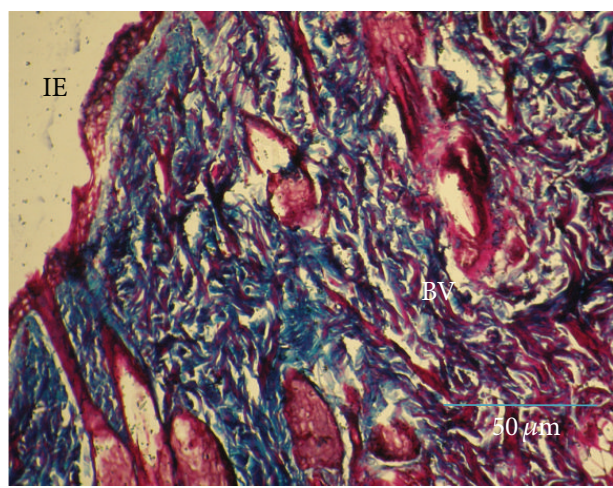

(a)

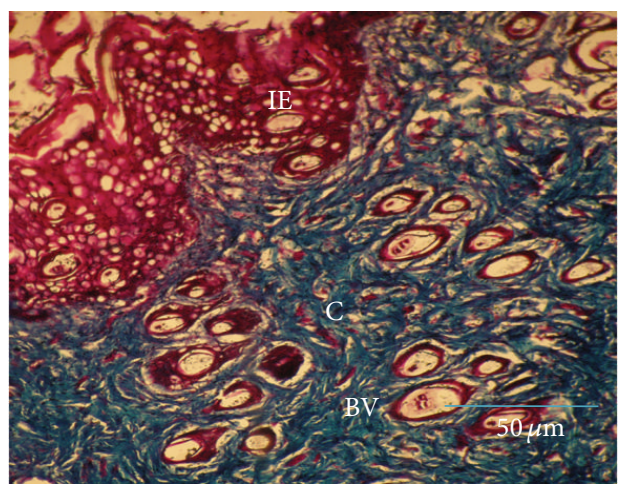

(c)

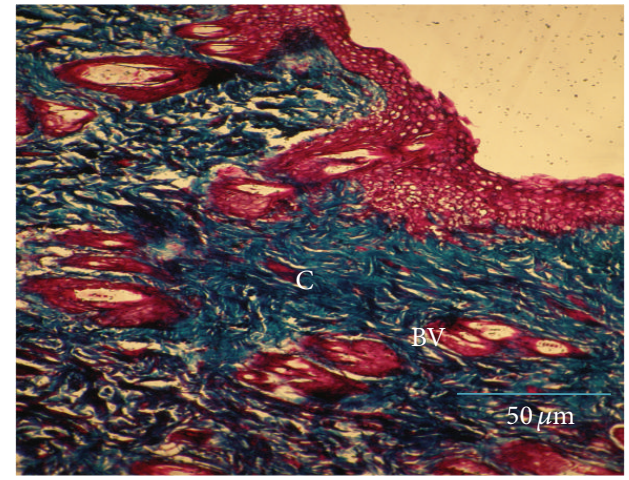

(b)

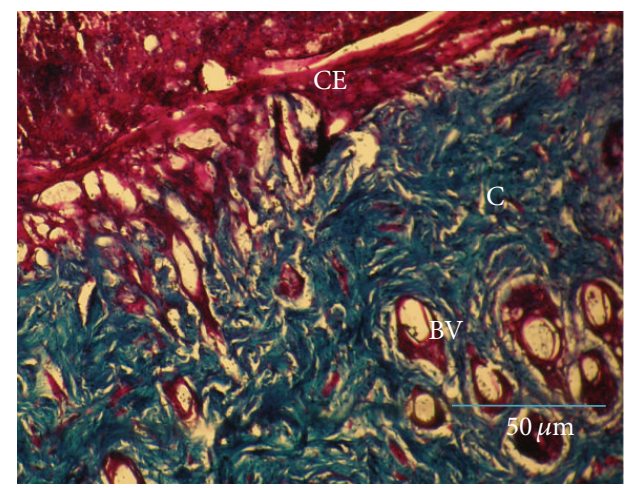

(d)

Figure 5: Masson's trichrome staining for collagen in control and A. squamosa treated wound tissues from first and second week, respectively (magnification 20x). Control (a) shows collagen fibers with incomplete epithelialization whereas (b) treated tissue shows dense collagen deposition. In the second week, control (c) depicts thin collagen layer at the wound site and (d) treated tissue shows complete epithelialization with uniform collagen deposition. IE: incomplete epithelialization, CE: complete epithelialization, E: epithelialization, BV: blood vessels, and C: collagen. Scale bar: $50 \mu \mathrm{m}$.

secrete hyaluronic acid into the extracellular matrix [35]. The role of hyaluronate gel in wound healing in streptozotocin induced diabetic rats has already been reported [36].

HA might be the rationale behind the availability of excess fluid and flexible matrix on the wound site that facilitates greater cell mobility and easier and faster regeneration of damaged tissue. The increased content of HA in A. squamosa treated wounds may result in the formation of a more stable scar [13]. Chondroitin sulfates $\mathrm{A}$ and $\mathrm{C}$ have a crucial role in in vitro proliferation of fibroblasts to accelerate the wound closure rapidly through their location of sulfation group [37]. Dermatan sulphate proteoglycans are closely associated with collagen fibers [38]. They influence the collagen fibril formation in vitro and may therefore contribute to the organization and strength of the collagen fibrillar assembly in wound tissues [39].

A significant reduction in the levels of keratan sulfate in the treated groups was observed. Funderburgh has reported that myofibroblasts exhibited reduced expression of keratocan, a keratan sulfate linked proteoglycan. The increased rate of contraction in the treated groups suggested that A. squamosa extract accelerates the transformation of fibroblasts to myofibroblasts. Reduced level of keratan sulfate in the wound tissue is associated with inflammation, suggesting the role of proinflammatory cytokines involved in the downregulation of keratan sulfate biosynthesis [40, 41]. Cintron et al. have shown that corneal wound healing resulted in a reduction of keratan sulfate and in accumulation of highly sulfated chondroitin/dermatan sulfate in the scar. The results we obtained also substantiate this observation [42].

Collagen, a principal component of connective tissue, plays a major role in the healing of wounds by providing a structural architecture for the remodeling tissue. Collagen molecules contain aldehydic groups that cross-link with amino acids to form collagen fibers. Normally, an increase in collagen synthesis would result in an increase in aldehyde content which leads to a greater potential for cross-linking $[43,44]$.

The present investigation shows that the collagen obtained from A. squamosa treated wounds has a higher content of aldehydic groups (Table 3) than collagen from controls. This observation indicates that the collagen in treated wounds must have undergone a greater degree of cross-linking resulting in an ultimate increase in wound 


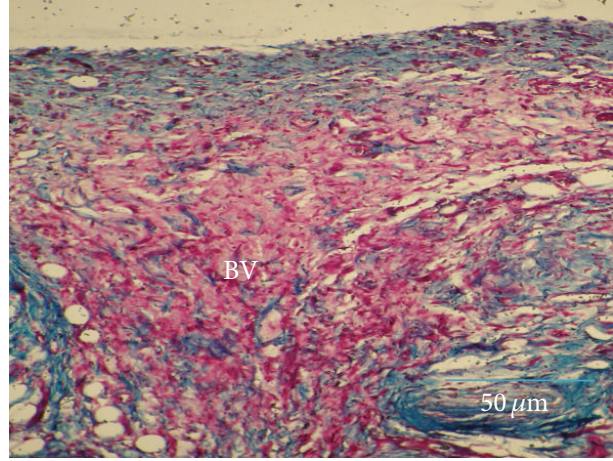

(a)

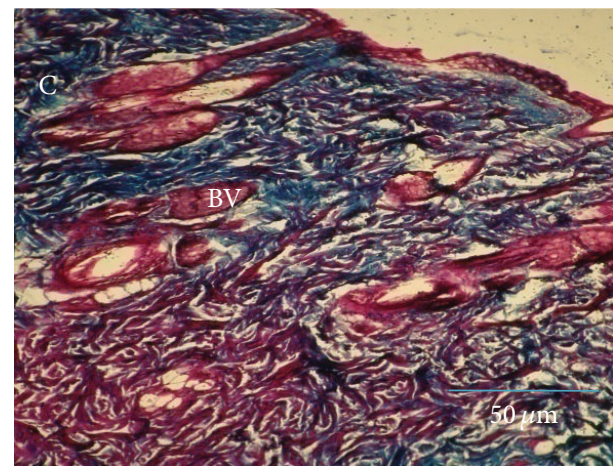

(c)

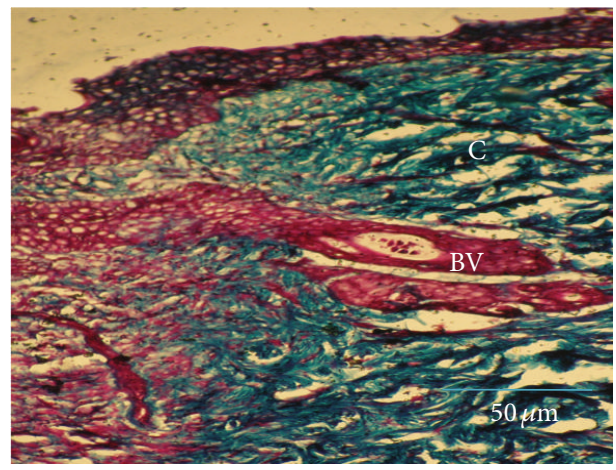

(e)

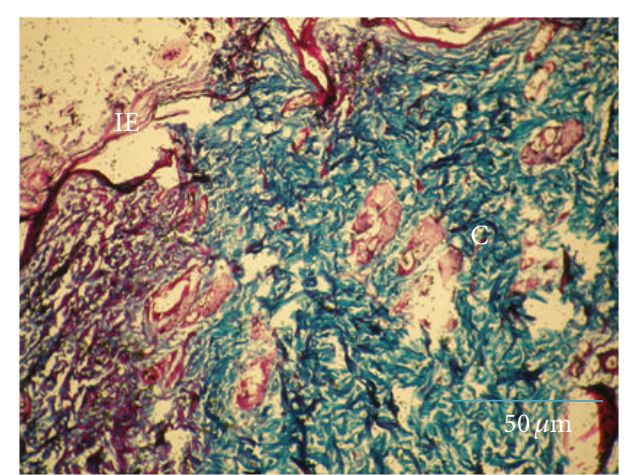

(b)

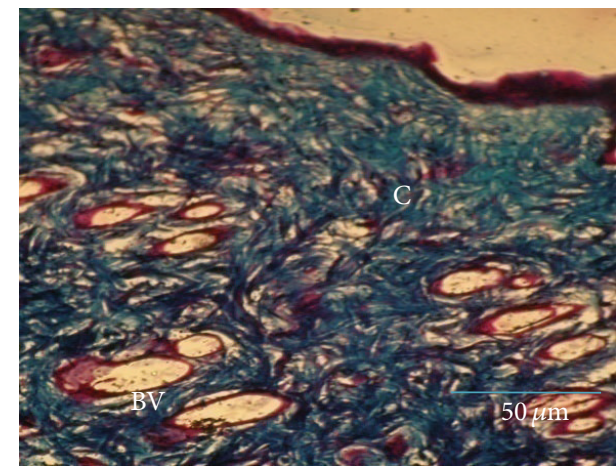

(d)

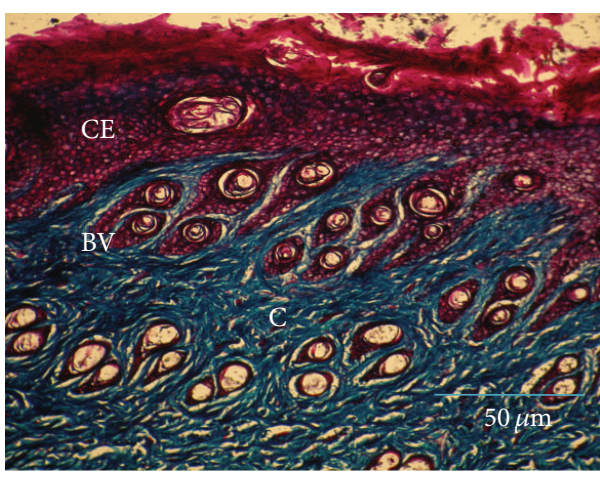

(f)

Figure 6: Masson's trichrome staining for collagen in control and A. squamosa treated diabetic wound tissues from first, second, and third week, respectively (magnification 20x). Control (a) shows large number of proliferating blood vessels, whereas (b) treated tissue shows less collagen formation. In the second week, control (c) depicts adequate amount of blood vessels formation and collagen fibers and (d) treated tissue shows thick collagen fiber deposition under thin epithelial layer. In the third week, control (e) shows accumulation of loosely arranged collagen bundles at the wound site and (f) explores the completed epithelial layer with highly organized collagen deposition. IE: incomplete epithelialization, E: epithelialization, C: collagen, and BV: blood vessels. Scale bar: $50 \mu \mathrm{m}$.

strength, which was further confirmed by the tensile strength of the wounds.

Solubility pattern of collagen in neutral salt buffer and acid solution mainly depends on the cross-linking of collagen. Highly cross-linked collagen becomes less soluble in neutral salt buffer and acid solution which can be released only in pepsin digestion. A. squamosa treatment showed decreased percentage of solubility in neutral buffer and in dilute acid solution. A significantly larger amount was solubilized in pepsin digestion and this is an indication of increased levels of cross-linking in treated groups. The insoluble collagen content of treated groups is also higher than that of the control group.

Type I collagen is the most abundant type of collagen observed in normal dermis (approximately $80 \%$ to $90 \%$ ). But during the early phases of wound healing, fibroblasts actively produce type III collagen, which may account for 30\% of the total collagen in a healing wound. By week 2, type I collagen again becomes the principal collagen produced by fibroblasts. Type I and type III collagen are formed in skin in 
a higher proportion relative to other types and are maintained in a fixed proportion relative to one another in normal skin tissue [45]. A. squamosa treated wounds synthesize greater amounts of type III collagen when compared to controls. The presence of higher levels of type III collagen may have beneficial effects on early wound healing process and result in better organization of type I collagen in the final scar [32].

Histological evaluations using specific stains strongly support these results. A greater degree of epithelialization and collagen deposition observed in A. squamosa treated wounds reveals the healing efficacy of the plant extract. Proliferated blood capillaries and dense collagen fibers were uniformly distributed, significantly increased at the wound site in normal treated and diabetic treated rats, especially during second and third week, respectively. These changes indicate a shift in wound healing from the proliferative phase to the maturation phase.

Earlier, we have reported that the rate of wound contraction was significantly higher and period of epithelialization was shorter in normal and diabetic treated rats, when treated with $A$. squamosa [15]. These results further strongly substantiate the efficacy of $A$. squamosa in collagen maturation during wound repair.

\section{Conclusion}

We have shown that the wound healing efficacy of $A$. squamosa in human dermal fibroblast by assessing cell proliferation and wound closure through scratch wound assay and the topical administration of ethanolic extract of $A$. squamosa promotes active synthesis of GAGs and collagen maturation during wound healing in normal and diabetic rats. This in turn is a positive sign of better wound healing.

\section{Conflict of Interests}

The authors declare that there is no conflict of interests regarding the publication of this paper.

\section{Acknowledgments}

The authors thank the director of CLRI for the facility provided. T. Ponrasu acknowledges the Council of Scientific and Industrial Research (CSIR), New Delhi, for the Award of Senior Research Fellowship. The authors wish also to thank Dr. T. Narasimhaswamy for his help in microscopical analyses.

\section{References}

[1] V. Falanga, "Wound healing and its impairment in the diabetic foot," The Lancet, vol. 366, no. 9498, pp. 1736-1743, 2005.

[2] W. K. Stadelmann, A. G. Digenis, and G. R. Tobin, "Physiology and healing dynamics of chronic cutaneous wounds," The American Journal of Surgery, vol. 176, 1, no. 2, pp. 26S-38S, 1998.

[3] B. Kumar, M. Vijayakumar, R. Govindarajan, and P. Pushpangadan, "Ethnopharmacological approaches to wound healingexploring medicinal plants of India," Journal of Ethnopharmacology, vol. 114, no. 2, pp. 103-113, 2007.
[4] Y. Iba, A. Shibata, M. Kato, and T. Masukawa, "Possible involvement of mast cells in collagen remodeling in the late phase of cutaneous wound healing in mice," International Immunopharmacology, vol. 4, no. 14, pp. 1873-1880, 2004.

[5] A. B. Wysocki, "Wound fluids and the pathogenesis of chronic wounds," Journal of Wound, Ostomy and Continence Nursing, vol. 23, no. 6, pp. 283-290, 1996.

[6] R. N. Nancy Broderick, "Understanding chronic wound healing," Journal for Nurse Practitioners, vol. 34, no. 10, pp. 16-22, 2009.

[7] R. V. Iozzo, "Matrix proteoglycans: from molecular design to cellular function," Annual Review of Biochemistry, vol. 67, pp. 609-652, 1998

[8] K. G. Vogel and J. A. Trotter, "The effect of proteoglycans on the morphology of collagen fibrils formed in vitro," Collagen and Related Research, vol. 7, no. 2, pp. 105-114, 1987.

[9] J. M. Trownbridge and R. L. Gallo, "Dermatan sulfate: new functions from an old glycosaminoglycan," Glycobiology, vol. 12, no. 9, pp. 117R-125R, 2002.

[10] M. J. Reed, P. Puolakkainen, T. F. Lane, D. Dickerson, P. Bornstein, and E. H. Sage, "Differential expression of SPARC and thrombospondin 1 in wound repair: immunolocalization and in situ hybridization," Journal of Histochemistry and Cytochemistry, vol. 41, no. 10, pp. 1467-1477, 1993.

[11] J. F. Bateman, S. R. Lamande, and J. A. M. Ramshaw, "Collagen superfamily," in Extracellular Matrix. Vol. 2 Molecular Components and Interactions, W. D. Comper, Ed., pp. 22-67, Harwood Academic Publishers, Amsterdam, The Netherlands, 1996.

[12] S. Guo and L. A. DiPietro, "Factors affecting wound healing," Journal of Dental Research, vol. 89, no. 3, pp. 219-229, 2010.

[13] P. Chithra, G. B. Sajithlal, and G. Chandrakasan, "Influence of Aloe vera on the glycosaminoglycans in the matrix of healing dermal wounds in rats," Journal of Ethnopharmacology, vol. 59, no. 3, pp. 179-186, 1998.

[14] M. Sumitra, P. Manikandan, V. S. Gayathri, P. Mahendran, and L. Suguna, "Emblica officinalis exerts wound healing action through up-regulation of collagen and extracellular signalregulated kinases (ERK1/2)," Wound Repair and Regeneration, vol. 17, no. 1, pp. 99-107, 2009.

[15] T. Ponrasu and L. Suguna, "Efficacy of Annona squamosa on wound healing in streptozotocin-induced diabetic rats," International Wound Journal, vol. 9, no. 6, pp. 613-623, 2012.

[16] T. Mosmann, "Rapid colorimetric assay for cellular growth and survival: application to proliferation and cytotoxicity assays," Journal of Immunological Methods, vol. 65, no. 1-2, pp. 55-63, 1983.

[17] F. Kheradmand, H. G. Folkesson, L. Shum, R. Derynk, R. Pytela, and M. A. Matthay, "TGF $\alpha$ enhances alveolar epithelial cell repair in a new in vitro model," The American Journal of Physiology, vol. 267, no. 6, pp. L728-L738, 1994.

[18] N. Rakieten, M. L. Rakieten, and M. R. Nadkarni, "Studies on the diabetogenic action of streptozotocin (NSC-37917)," Cancer Chemotherapy Reports, vol. 29, pp. 91-98, 1963.

[19] P. Masiello, C. Broca, R. Gross et al., "Experimental NIDDM: development of a new model in adult rats administered streptozotocin and nicotinamide," Diabetes, vol. 47, no. 2, pp. 224-229, 1998.

[20] I. Mackraj, S. Ramesar, M. Singh et al., "The in vivo effects of Tulbhagia violacea on blood pressure in a salt-sensitive rat model," Journal of Ethnopharmacology, vol. 117, no. 2, pp. 263269, 2008. 
[21] R. L. Smith, E. Gilkerson, N. Kohatsu, T. Merchant, and D. J. Schurman, "Quantitative microanalysis of synovial fluid and articular cartilage glycosaminoglycans," Analytical Biochemistry, vol. 103, no. 1, pp. 191-200, 1980.

[22] S. Schiller, G. Slover, and A. A. Dorfman, "A new perspective with particular reference to ascorbic acid deficiency," The Journal of Biological Chemistry, vol. 36, pp. 983-985, 1961.

[23] M. Breen, P. A. Knepper, H. G. Weinstein, L. J. Blacik, D. G. Lewandowski, and B. M. Baltrus, "Microanalysis of glycosaminoglycans," Analytical Biochemistry, vol. 113, no. 2, pp. 416-422, 1981.

[24] K. A. Piez, "The amino acid chemistry of some calcified tissues," Annals of the New York Academy of Sciences, vol. 109, pp. 256268, 1963 .

[25] M. Adam, P. Fietzek, and K. Kühn, "Investigations on the reaction of metals with collagen in vivo. 2. the formation of cross-links in the collagen of lathyritic rats after gold treatment in vivo," European Journal of Biochemistry, vol. 3, no. 4, pp. 411414, 1968.

[26] M. A. Paz, O. O. Blumenfeld, M. Rojkind, E. Henson, C. Furfine, and P. M. Gallop, "Determination of carbonyl compounds with N-methyl benzothiazolone hydrazone," Archives of Biochemistry and Biophysics, vol. 109, no. 3, pp. 548-559, 1965.

[27] T. M. Hering, R. E. Marchant, and J. M. Anderson, “Type V collagen during granulation tissue development," Experimental and Molecular Pathology, vol. 39, no. 2, pp. 219-229, 1983.

[28] A. E. Loo and B. Halliwell, "Effects of hydrogen peroxide in a keratinocyte-fibroblast co-culture model of wound healing," Biochemical and Biophysical Research Communications, vol. 423, no. 2, pp. 253-258, 2012.

[29] H. S. Dua, J. A. Gomes, and A. Singh, "Corneal epithelial wound healing," The British Journal of Ophthalmology, vol. 78, no. 5, pp. 401-408, 1994.

[30] J. Li, J. Chen, and R. Kirsner, "Pathophysiology of acute wound healing," Clinics in Dermatology, vol. 25, no. 1, pp. 9-18, 2007.

[31] R. L. Gallo and M. Bernfield, "Proteoglycan and their role in wound repair," in The Molecular and Cellular Biology of Wound Repair, R. A. F. Clark, Ed., pp. 475-492, Plenum Press, New York, NY, USA, 1996.

[32] P. Chithra, G. B. Sajithlal, and G. Chandrakasan, "Influence of Aloe vera on collagen characteristics in healing dermal wounds in rats," Molecular and Cellular Biochemistry, vol. 181, no. 1-2, pp. 71-76, 1998.

[33] R. Moseley, R. J. Waddington, and G. Embery, "Hyaluronan and its potential role in periodontal healing," Dental Update, vol. 29, no. 3, pp. 144-148, 2002.

[34] B. A. Mast, R. F. Diegelmann, T. M. Krummel, and I. K. Cohen, "Hyaluronic acid modulates proliferation, collagen and protein synthesis of cultured fetal fibroblasts," Matrix, vol. 13, no. 6, pp. 441-446, 1993.

[35] F. L. Mesa, J. Aneiros, A. Cabrera et al., "Antiproliferative effect of topic hyaluronic acid gel: study in gingival biopsies of patients with periodontal disease," Histology and Histopathology, vol. 17, no. 3, pp. 747-753, 2002.

[36] F. Al Bayaty, M. Abdulla, M. I. Abu Hassan, and M. Masud, "Wound healing potential by hyaluronate gel in streptozotocininduced diabetic rats," Scientific Research and Essays, vol. 5, no. 18, pp. 2756-2760, 2010.

[37] X. H. Zou, Y. Z. Jiang, G. R. Zhang, H. M. Jin, T. M. Nguyen, and H. W. Ouyang, "Specific interactions between human fibroblasts and particular chondroitin sulfate molecules for wound healing," Acta Biomaterialia, vol. 5, no. 5, pp. 1588-1595, 2009.

[38] R. Fleischmajer, L. W. Fisher, E. D. MacDonald, L. Jacobs Jr., J. S. Perlish, and J. D. Termine, "Decorin interacts with fibrillar collagen of embryonic and adult human skin," Journal of Structural Biology, vol. 106, no. 1, pp. 82-90, 1991.

[39] J. E. Scott, "Proteoglycan-fibrillar collagen interactions," Biochemical Journal, vol. 252, no. 2, pp. 313-323, 1988.

[40] J. L. Funderburgh, "Keratan sulfate biosynthesis," IUBMB Life, vol. 54, no. 4, pp. 187-194, 2002.

[41] M. Rodrigues, V. Nirankari, S. Rajagopalan, K. Jones, and J. Funderburgh, "Clinical and histopathologic changes in the host cornea after epikeratoplasty for keratoconus," The American Journal of Ophthalmology, vol. 114, no. 2, pp. 161-170, 1992.

[42] C. Cintron, J. D. Gregory, S. P. Damle, and C. L. Kublin, "Biochemical analyses of proteoglycans in rabbit corneal scars," Investigative Ophthalmology and Visual Science, vol. 31, no. 10, pp. 1975-1981, 1990.

[43] R. C. Siegel, "Collagen cross linking: synthesis of collagen cross links in vitro with highly purified lysyl oxidase," The Journal of Biological Chemistry, vol. 251, no. 18, pp. 5786-5792, 1976.

[44] M. L. Tanzer, "Cross-linking," in Biochemistry of Collagen, G. N. Ramachandran and A. H. Reddi, Eds., pp. 137-162, Plenum Press, New York, NY, USA, 1976.

[45] W. Cheng, R. Yan-Hua, N. Fang-Gang, and Z. Guo-An, "The content and ratio of type I and III collagen in skin differ with age and injury," African Journal of Biotechnology, vol. 10, no. 13, pp. 2524-2529, 2011. 

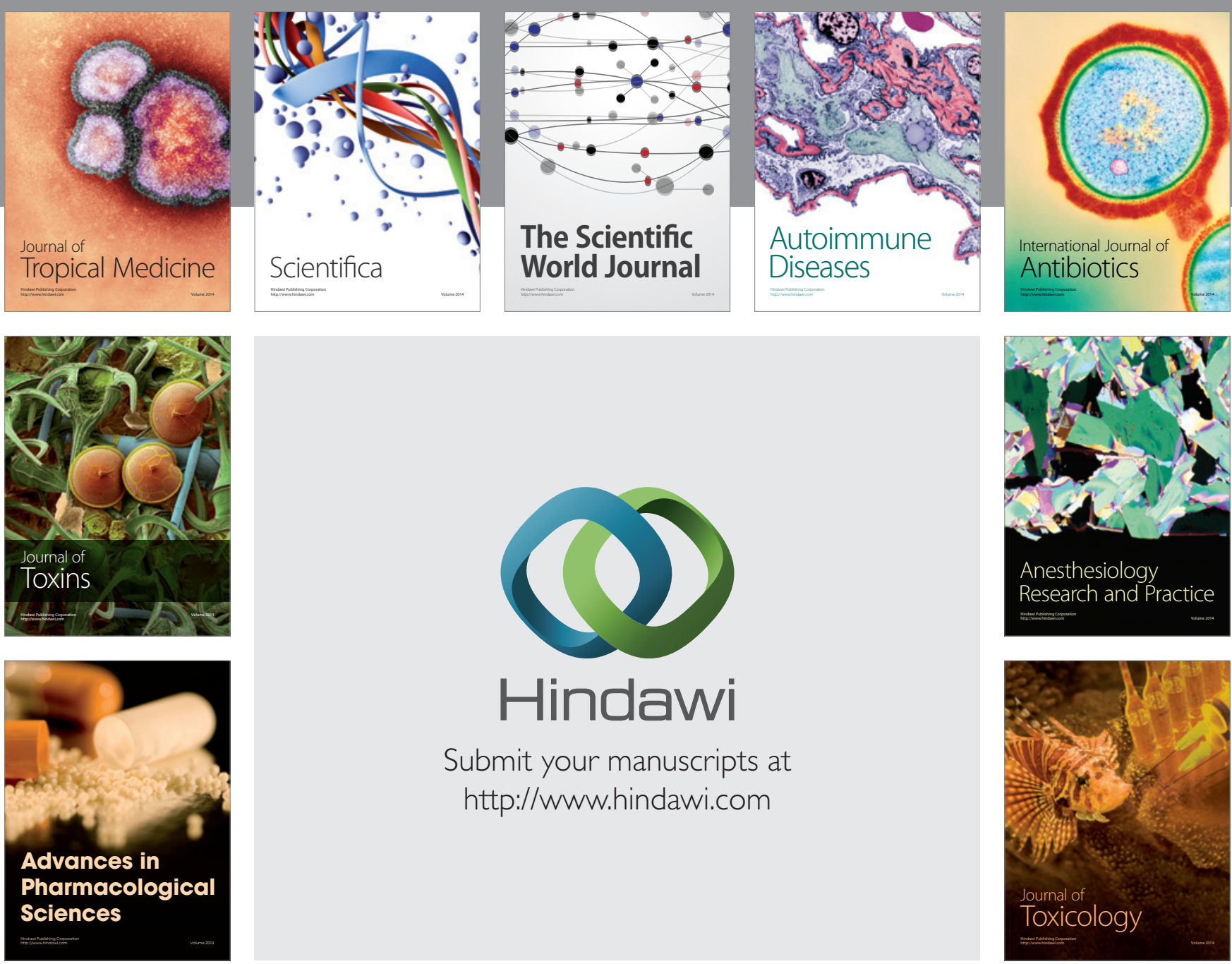

\section{Hindawi}

Submit your manuscripts at

http://www.hindawi.com
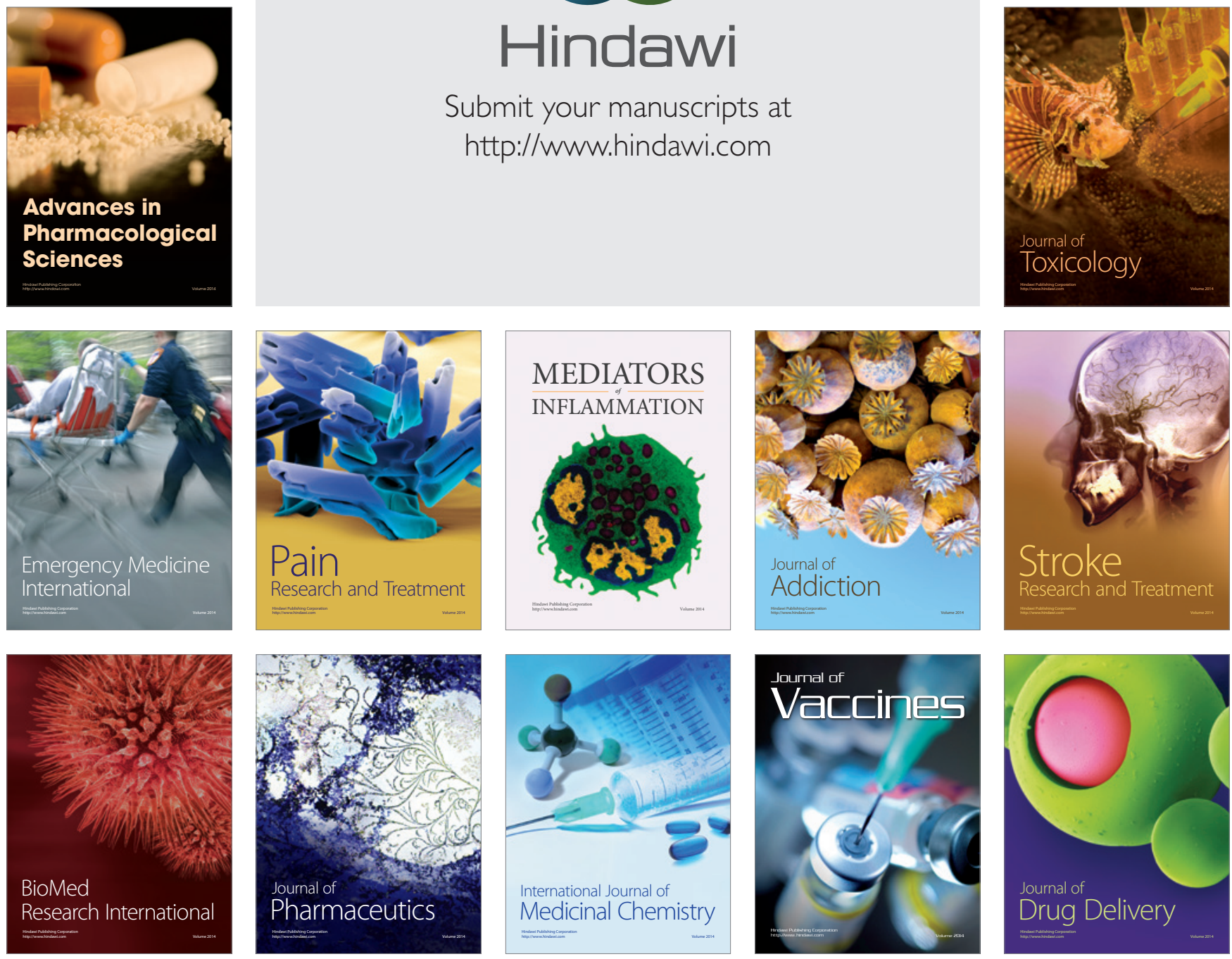